July 24, 1998

\title{
Quantum energy flow, dissipation and decoherence in mesoscopic dielectric structures
}

\author{
M. P. Blencowe \\ The Blackett Laboratory, Imperial College, London SW7 $2 B Z$
}

\begin{abstract}
We first present a summary of recent results concerning the phononic energy transport properties of mesoscopic, suspended dielectric wires. We then discuss some related open problems concerning the fundamental lower limits on the vibrational damping rates of submicron-sized cantilever structures and also the possibility to create and detect quantum superpositions of spatially separated states for such structures.
\end{abstract}

Keywords: quantum wires, Landauer thermal conductance, mesoscopic mechanical dissipation, quantum decoherence

Address for correspondence: M. P. Blencowe, The Blackett Lab., Imperial College, London SW7 2BZ, England. Fax: +44-171-594-7604. E-mail: m.blencowe@ic.ac.uk 
Recent experiments by Michael Roukes and coworkers on heat transport in submicronsized, suspended dielectric wires [1] have opened up new avenues for investigation in the fields of mesoscopics and phonon physics. Just as for electrons in conventional quantum wells, wires etc., phonons confined to the suspended wires can exhibit manifestly nonclassical behaviour. For example, the low temperature thermal conductance is quantized in universal units $\pi k_{B}^{2} T / 6 \hbar$, analogous to the well-known $2 e^{2} / h$ electronic conductance quantum [2 《. This result follows from the Landauer formula for the thermal conductance:

$$
\kappa=\frac{\pi k_{B}^{2} T}{6 \hbar} \sum_{n, n^{\prime}} \int_{\frac{E_{n, 0}}{k_{B} T}}^{\infty} d \epsilon g(\epsilon) T_{n^{\prime} n}^{21}\left(\epsilon k_{B} T\right),
$$

where $T$ is the average temperature of the two phonon reservoirs (with the reservoir temperature difference supposed small), $T_{n^{\prime} n}^{21}(E)$ is the probability for a phonon with energy $E$ in subband $n$ of lead 1 to be (elastically) transmitted into subband $n^{\prime}$ of lead 2 , and $E_{n, 0}$ is the zone-centre energy of subband $n$. The function $g(\epsilon)$ is defined as follows:

$$
g(\epsilon)=\frac{3 \epsilon e^{\epsilon}}{\pi^{2}\left(e^{\epsilon}-1\right)^{2}}
$$

and satisfies $\int_{0}^{\infty} d \epsilon g(\epsilon)=1$. Thus, in the absence of scattering, i.e. $T_{n^{\prime} n}^{21}=\delta_{n^{\prime} n}$, a given subband $n$ contributes to the reduced conductance $\kappa / T$ the universal quantum $\pi k_{B}^{2} / 6 \hbar \approx 9.465 \times 10^{-13} \mathrm{WK}^{-2}$ in the limit $E_{n, 0} / k_{B} T \rightarrow 0$. In Fig. 1, we show the temperature dependence of the reduced thermal conductance for perfect GaAs wires with uniform, rectangular cross sections of various dimensions comparable to those used in the experiments of Ref. [1]. The only wire characteristics which are needed in order to determine the conductance are the zone-center frequencies $\omega_{n, 0}$. These can be calculated using the elegant numerical method developed in Ref. [5]. Note that there are no steplike features, a consequence of the broadness of the Bose-Einstein distribution as compared with the zone-centre energy separations. There is, however, a plateau for $T \rightarrow 0$ where only phonons in the lowest subband with $E_{n, 0}=0$ contribute. The plateau has the value four in universal quantum units, a consequence of there being four basic mode types: dilatational, torsional and two types of flexural mode. 
The devices of Roukes and coworkers are in principle capable of measuring much more than just the thermal conductance. Because of the very small volume and hence heat capacity of the integrated electron gas thermometers, adsorption and emission of single phonons can produce measurable fluctuations in the gas temperature. From the magnitude of a given temperature fluctuation, the energy of the absorbed or emitted phonon is known and, thus, there is the possibility for high resolution phonon spectroscopy, one of the "holy grails" of the phonon physics community. In particular, by measuring the fluctuation statistics for the absorption of phonons in a given narrow energy interval, information can be obtained concerning the energy dependence of the phonon transmission probability for the suspended wires and also of the electron-phonon interaction in the wires. An initial discussion can be found in Ref. [4].

We finish this note with a brief mention of two open problems which are somewhat related to the above discussion. The first concerns the vibrational damping rates of submicron-sized, single crystal cantilevers. In such small structures, volume defects are rare and preliminary experiments suggest that excitation of surface defects provides the dominant damping mechanism [6.7]. However, the presence and nature of surface defects is strongly dependent on the cantilever fabrication methods, so that appropriate modifications in these methods will lead to reduced surface defect densities. A natural question then is the following: what is the lowest possible damping rate which can be achieved for a given cantilever vibrational mode? In a defect-free, single crystal dielectric cantilever, only two dissipation mechanisms remain: 1) the coupling of the cantilever vibrational modes to the substrate modes at the cantilever base, and 2) the coupling via the anharmonic interaction of the vibrational modes to the background thermal phonon distribution in the cantilever. These mechanisms are unavoidable and thus set a fundamental lower limit on the damping rate. Submicron-sized cantilevers can have fundamental flexural frequencies ranging from $\mathrm{KHz}$ to $\mathrm{GHz}$ and it may be the case that the relative strengths of the two mechanisms varies considerably over this range. Just as for the energy flow properties, it is expected that submicron-sized structures with dimensions comparable to the thermal phonon wavelength will exhibit qualitatively 
different fundamental damping behaviour from that of bulk mechanical oscillators. It would be interesting to determine the relative strengths of the fundamental damping mechanisms. They are well-characterised at the microscopic level and so should be accessible to theoretical analysis.

The second problem concerns the possibility of creating and detecting quantum superpositions of spatially separated states for submicron-sized vibrating cantilevers. In the field of quantum optics, Haroche and coworkers have successfully demonstrated the creation and detection of superpositions of quantum states involving radiation fields [8]. A recent theoretical investigation by the Quantum Optics Group at Imperial College [9] has suggested that such field states can be used to drive a submicron-sized, moveable mirror into a quantum superposition of spatially separated states. Such a quantum superposition will typically decohere extremely rapidly into a classical mixture state as a result of dissipation. However, their estimates suggest that the decoherence rates of submicron-sized moveable mirrors fabricated using similar methods to those of Roukes' group will be sufficiently low so as to be able to observe the quantum superpositions. In the light of this possibility, a natural question for a condensed matter physicist concerns whether an electron current could be used to drive a submicron-sized cantilever into a nonclassical state. One possible device is shown in Fig. 2. A moveable quantum dot is located between two quantum wire contacts. Nanometer-scale gaps separate the quantum dot from the wire contacts, so that an electron can only move from one contact to the other via the quantum dot by tunneling across the gaps. When an electron is in the quantum dot it can impart some of its momentum to the dot, causing it to move, whereas when the electron is in one of the contacts it has no influence on the dot motion. Thus, the quantum uncertainty in a tunneling electron's location will induce some uncertainty in the dot's motion. It would be interesting to determine possible signatures in the measured electron current of this quantum uncertainty in the dot motion. 


\section{ACKNOWLEDGMENTS}

The author would like to thank the organizers of Phonons 98 for providing the oppor-

tunity to present this work. Funding by the EPSRC under Grant No. GR/K/55493 is also acknowledged. 


\section{REFERENCES}

[1] T. S. Tighe, J. M. Worlock, and M. L. Roukes, Appl. Phys. Lett. 70, 2687 (1997).

[2] D. E. Angelescu, M. C. Cross, and M. L. Roukes, Superlattices and Microstructures 23, $673(1998)$.

[3] L. G. C. Rego and G. Kirczenow, Phys. Rev. Lett. 81, 232 (1998).

[4] M. P. Blencowe, cond-mat/9803319 and Phys. Rev. B (to be published).

[5] N. Nishiguchi, Y. Ando, and M. N. Wybourne, J. Phys.: Condens. Matter 9, 5751 (1997).

[6] D. A. Harrington and M. L. Roukes, Bull. Am. Phys. Soc. 43,405 (1998).

[7] K. Yasumura et al., Bull. Am. Phys. Soc. 43, 370 (1998).

[8] M. Brune et al., Phys. Rev. Lett. 77, 4887 (1996).

[9] S. Bose, K. Jacobs and P. L. Knight, quant-ph/9712021 (unpublished). 


\section{FIGURES}

FIG. 1. Reduced thermal conductance in universal units versus temperature for perfect GaAs wires with uniform rectangular cross sections $200 \mathrm{~nm} \times 400 \mathrm{~nm}$ (solid line), $200 \mathrm{~nm} \times 300 \mathrm{~nm}$ (dashed line) and $200 \mathrm{~nm} \times 100 \mathrm{~nm}$ (dotted line).

FIG. 2. Model single electron potential profile for a movable quantum dot located between two quantum wire contacts. 


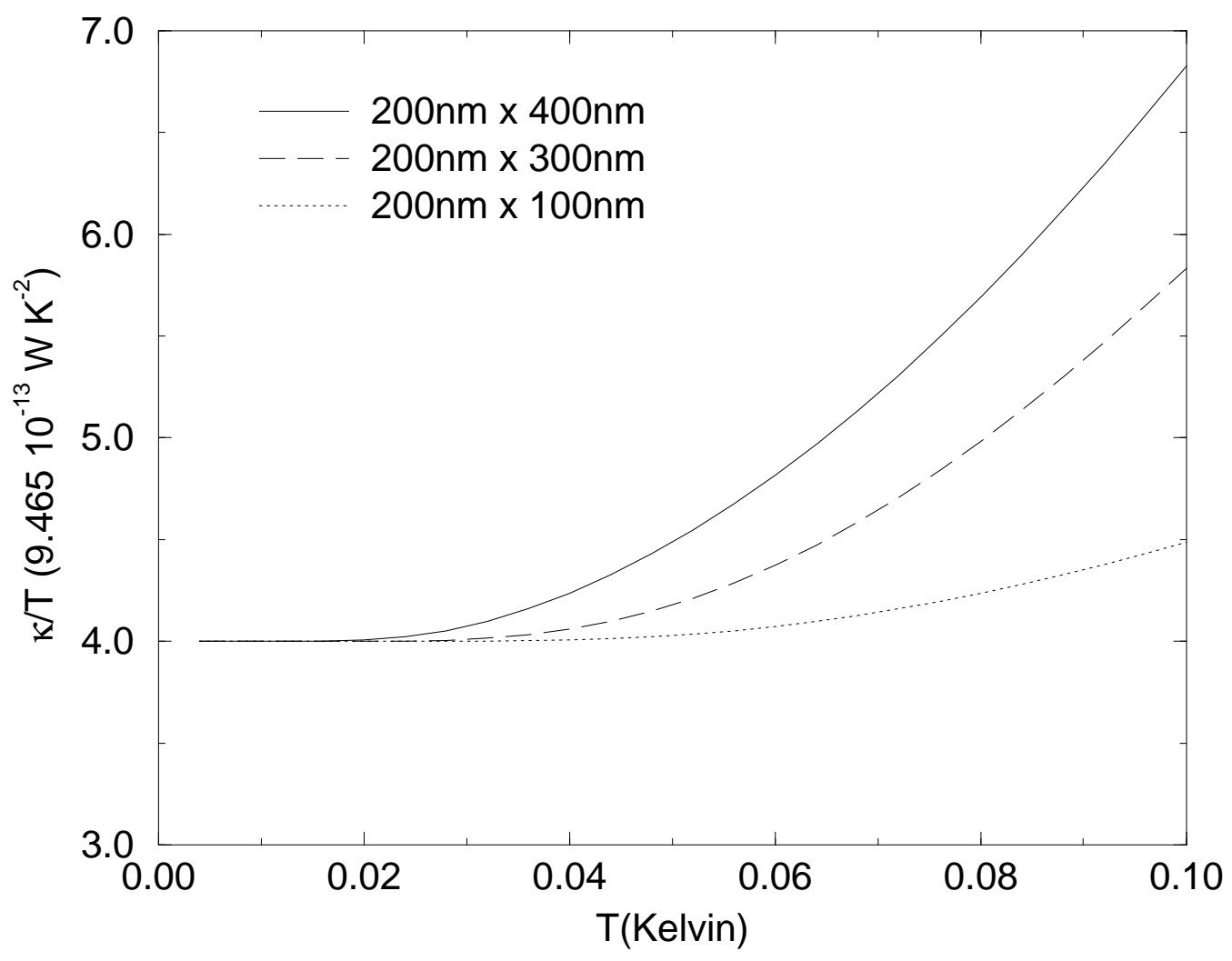

FIG. 1. 

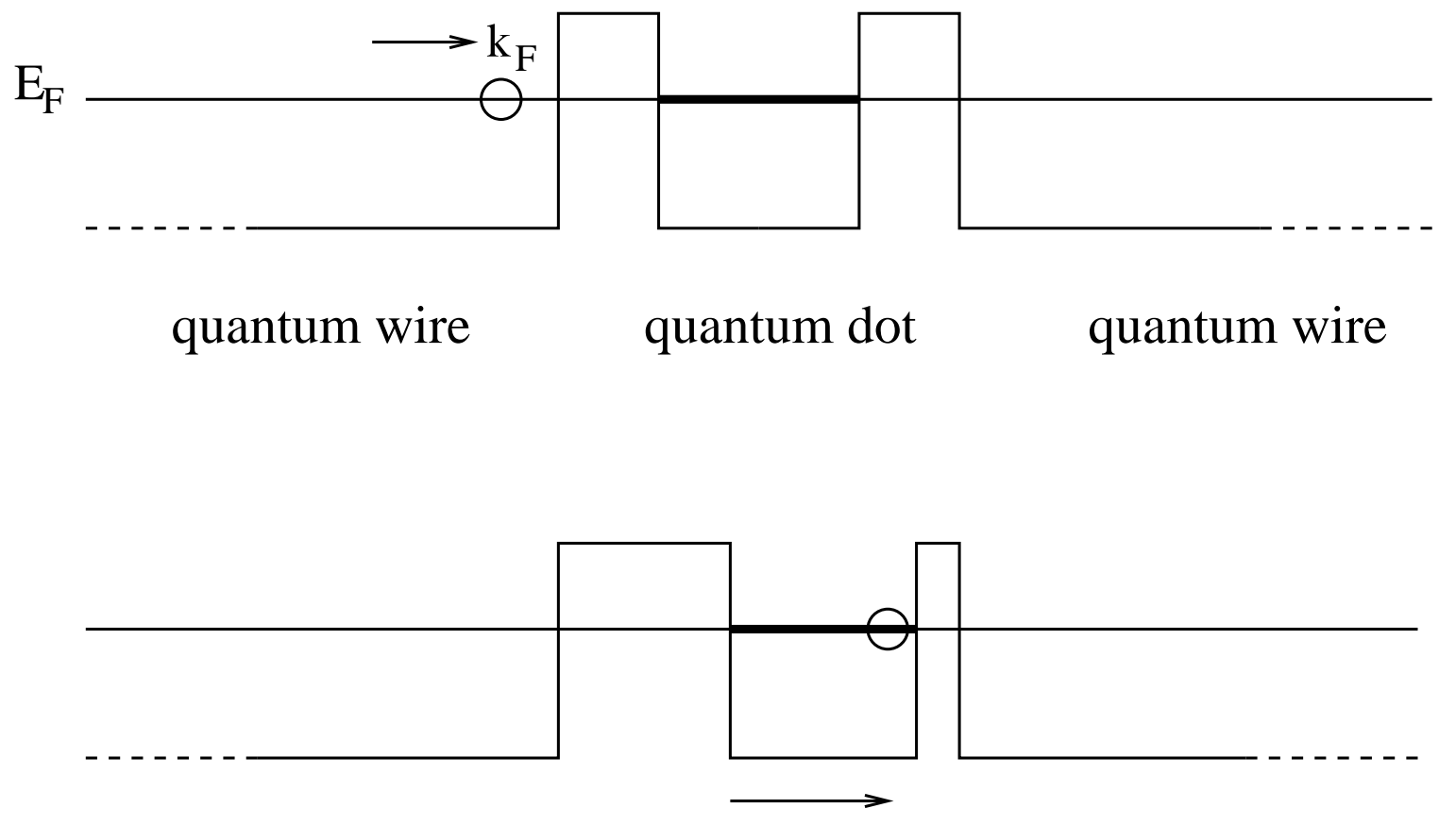

FIG. 2. 